\title{
On the notion of boundary conditions in comparison principles for viscosity solutions
}

\author{
Max Jensen and Iain Smears
}

\begin{abstract}
We collect examples of boundary-value problems of Dirichlet and Dirichlet-Neumann type which we found instructive when designing and analysing numerical methods for fully nonlinear elliptic partial differential equations. In particular, our model problem is the Monge-Ampère equation, which is treated through its equivalent reformulation as a HamiltonJacobi-Bellman equation. Our examples illustrate how the different notions of boundary conditions appearing in the literature may admit different sets of viscosity sub- and supersolutions. We then discuss how these examples relate to the application of comparison principles in the analysis of numerical methods.
\end{abstract}

Keywords. Viscosity boundary conditions, comparison principles, Hamilton-Jacobi-Bellman equations, Monge-Ampère equations, Barles-Souganidis theorem.

AMS classification. 49L25, 65N99.

\section{Introduction}

In this short note we collect a small number of examples which we found instructive when designing and analysing numerical methods for fully nonlinear elliptic partial differential equations (PDE). In particular, we are interested in the comparison principle between sub- and supersolutions, as used in the convergence proof by Barles and Souganidis [4] for the approximation of viscosity solutions by monotone numerical schemes. Recall that the comparison principle required for the analysis [4, Eq. (2.5)], called there the strong uniqueness property, was stated as an assumption.

Our model problem is the following simple Monge-Ampère equation

$$
M\left(D^{2} u\right)=0, \quad M(A):=\frac{1}{2} f^{2}-\operatorname{det} A
$$

on a domain $\Omega \subset \mathbb{R}^{d}, d \geq 2$ with $f \geq 0$.

The problem is complemented with either Dirichlet or mixed Dirichlet-Neumann boundary conditions, as well as the requirement that $u$ be a convex function in $\Omega$. In order to conform to the standard framework of degenerate elliptic operators, we consider the following reformulation of (1.1) as a Hamilton-Jacobi-Bellman (HJB) equation $[11,8]$ 


$$
H\left(D^{2} u\right)=0, \quad H(A):=\sup _{B \in \mathcal{S}_{1}}(-B: A+f \sqrt{\operatorname{det} B}),
$$

where $\mathcal{S}_{1}$ is the set of symmetric positive semidefinite matrices in $\mathbb{R}^{d \times d}$ with trace equal to 1 and $B: A:=\operatorname{trace}(B \cdot A)$. In particular, it was shown [8] that (1.1) (including the convexity constraint) is equivalent to (1.2) in the sense of viscosity solutions.

Note that this equivalence result concerns only the equations inside the domain $\Omega$, without referring to the boundary condition.

The reason for selecting this as our model problem is not only its relevance to applications, but also its degenerate elliptic structure, which will be exploited in the examples below. A further property of Dirichlet boundary conditions in combination with the Monge-Ampère operator is that the existence of solutions admitting classical boundary conditions depends on the convexity properties of the domain, making it an interesting test problem to explore different notions of Dirichlet boundary conditions.

Remark 1.1. Observe that the Barles-Souganidis theorem cannot be considered directly for (1.1) because (1.1) is only elliptic on the set of convex functions and its test functions are usually assumed to be convex [9, Definition 1.3.1]. This is the reason why we shall work with the equivalent formulation (1.2).

Comparison principles are central to the theory of viscosity solutions, both for the analysis of well-posedness of the PDE and for the analysis of numerical methods. While conceptually the statement of a comparison principle requires that subsolutions lie below supersolutions, the different formulations of the boundary conditions and the different sets of available test functions raise the question of the validity of the corresponding comparison principle. For instance, the boundary conditions can be imposed in the following variety of ways:

(i) In the classical sense, where the Dirichlet boundary condition is understood pointwise everywhere on the boundary; this is the setting for the comparison principle of Theorem 3.3 in the User's Guide [5] by Crandall, Ishii and Lions, and also in the numerical analysis in [8].

(ii) As in the setting of the Barles-Souganidis theorem [4], where the Dirichlet boundary condition is relaxed from its classical pointwise sense, and is understood in a generalised sense that allows extensions of the PDE onto the boundary. This notion of the boundary conditions is the subject of section 2 below.

(iii) As in Definition 7.4 of the User's Guide [5], where boundary conditions are relaxed similarly to the Barles-Souganidis approach, but semi-continuity of suband supersolutions is assumed from the outset and a closure operation is applied 
to the second-order jets. See also [3], where the semi-continuity for sub- and supersolutions of Hamilton-Jacobi equations is imposed, but the closure of the jets is not introduced.

We also refer the reader to [5, Definition 7.1] on the intermediate notion of the boundary condition named therein as the strong viscosity sense.

The sets of sub- and supersolutions are usually chosen within

(a) the spaces $\operatorname{USC}(\bar{\Omega})$ of bounded upper semi-continuous functions and $\operatorname{LSC}(\bar{\Omega})$ of bounded lower semi-continuous functions,

(b) or within the function space $C(\bar{\Omega})$ of continuous functions,

(c) or, in the classical setting, within the function space $C(\bar{\Omega}) \cap C^{2}(\Omega)$ of twice continuously differentiable functions.

Here, we shall focus our attention on the semi-continuous case because this is the relevant one for the analysis of numerical methods, where only the semi-continuity of upper and lower envelopes of sequences of numerical solutions is known a priori. Nevertheless, it is worth observing that the existence of a comparison principle may well be conditional to further regularity or structure assumptions on the set of sub- and supersolutions.

We point to Section 7.C of [5] for a general discussion of the subject.

Using the simple Monge-Ampère equation as a reference problem, we show examples where the sets of viscosity sub- and supersolutions vary depending on the notion of boundary condition employed, i.e. the different notions of boundary condition do lead to different solution sets in some cases.

In turn this also informs us how a numerical convergence analysis may be approached.

While the purpose of this note is to illustrate the differences between the various notions of boundary conditions with concrete examples, we point out that there is a substantial body of literature which provides analytic insights into this question. Besides the contributions mentioned above we name as examples [2] and [12, 13].

While we consider in the subsequent text different notions of viscosity sub- and supersolutions, a function $u$ is always said to be a viscosity solution if it is simultaneously a viscosity subsolution and supersolution.

Given a function $v$ we denote its upper semi-continuous envelope by $v^{*}$ and its lower semi-continuous envelope by $v_{*}$, respectively. More precisely, for all $x \in \bar{\Omega}$,

$$
v^{*}(x):=\sup _{\substack{\left\{y_{n}\right\}_{n} \subset \bar{\Omega} \\ y_{n} \rightarrow x}} \limsup _{n \rightarrow \infty} v\left(y_{n}\right), \quad v_{*}(x):=\inf _{\substack{\left\{y_{n}\right\}_{n} \subset \bar{\Omega} \\ y_{n} \rightarrow x}} \liminf _{n \rightarrow \infty} v\left(y_{n}\right),
$$

where $\sup _{\left\{y_{n}\right\}_{n} \subset \bar{\Omega}, y_{n} \rightarrow x}$ denotes the supremum over the set of all sequences with values in $\bar{\Omega}$ which converge to $x$. 


\section{Dirichlet boundary conditions as in the Barles-Souganidis theorem}

Let $\Omega$ be a open subset of $\mathbb{R}^{d}$ and consider the model problem (1.2) with a homogeneous Dirichlet boundary condition $u=0$ on $\partial \Omega$. In line with Definition 1.1 and equations (1.8), (1.9) of [4], we say that a locally bounded function $v$ is a viscosity subsolution of the boundary value problem if

$$
F_{*}\left(D^{2} \phi(x), v^{*}(x), x\right) \leq 0
$$

for all $\phi \in C^{2}(\bar{\Omega})$ such that $v^{*}-\phi$ has a local maximum at $x \in \bar{\Omega}$, where $F_{*}$ denotes the lower semicontinuous envelope of $F$ defined by

$$
F_{*}(A, w, x)= \begin{cases}H(A) & : x \in \Omega, \\ \min \{H(A), w\} & : x \in \partial \Omega .\end{cases}
$$

Analogously, $v$ is a viscosity supersolution whenever

$$
F^{*}\left(D^{2} \phi(x), v_{*}(x), x\right) \geq 0
$$

for all $\phi \in C^{2}(\bar{\Omega})$ such that $v_{*}-\phi$ has a local minimum at $x \in \bar{\Omega}$, where $F^{*}$ is the upper semicontinuous envelope of $F$ given by

$$
F^{*}(A, w, x)= \begin{cases}H(A) & : x \in \Omega, \\ \max \{H(A), w\} & : x \in \partial \Omega .\end{cases}
$$

We consider in the following example the Monge-Ampère equation on possibly one of the simplest domains with a boundary, namely a $d$-dimensional half-space. In particular, let $\Omega=\mathbb{H}^{d}$, with $d \geq 2$, where $\mathbb{H}^{d}=\left\{x=\left(x_{1}, \ldots, x_{d}\right) \in \mathbb{R}^{d}, x_{1}>0\right\}$, and consider the problem (1.2) with vanishing source term $f=0$, corresponding to the degenerate elliptic case, complemented with homogeneous Dirichlet boundary conditions on $\partial \Omega=\left\{x=\left(x_{1}, \ldots, x_{d}\right) \in \mathbb{R}^{d}, x_{1}=0\right\}$. It is clear that the function $u \equiv 0$ is a viscosity solution of the problem in the sense of [4].

In fact, by noticing that the definition of viscosity solution used in [4] does not prescribe any conditions at infinity on the solution, it is clear that this problem does not admit a unique classical solution. However, what we shall show below is that different notions of the boundary condition on $\partial \Omega$ admit different sets of viscosity solutions, regardless of the behaviour at infinity.

Proposition 2.1. Let $d \geq 2$ and let $\Omega=\mathbb{H}^{d}$ as above. For a fixed but arbitrary constant $c>0$, let the locally bounded function $v_{c}$ be defined by $v_{c}(x)=0$ if $x \in \Omega$ and $v_{c}(x)=-c$ if $x \in \partial \Omega$.

Then $v_{c}$ is a viscosity solution of (1.2) in the sense of [4]. 
Proof. It follows from the definition of $v_{c}$ that $\left(v_{c}\right)^{*} \equiv 0$ identically in $\bar{\Omega}$, whereas $\left(v_{c}\right)_{*}=v$ in $\bar{\Omega}$ since $v_{c}$ is lower semi-continuous. It is thus clear that $v_{c}$ is a viscosity subsolution of the problem.

We now prove that the function $v_{c}$ is also a viscosity supersolution and hence a viscosity solution of the problem in the sense of [4]; in particular, we must show that (2.1) holds for all $\phi \in C^{2}(\bar{\Omega})$ such that $\left(v_{c}\right)_{*}-\phi$ has a local minimum at $x \in \bar{\Omega}$. It is clear that (2.1) is satisfied whenever $x \in \Omega$ is an interior point, since $v_{*} \equiv 0$ in $\Omega$. Hence we need only to consider boundary points $x \in \partial \Omega$. Suppose now that $\phi \in C^{2}(\bar{\Omega})$ is such that $\left(v_{c}\right)_{*}-\phi$ has a local minimum at $x \in \partial \Omega$. Then, since $d \geq 2$, we may take a unit tangent vector $y=\left(0, y_{1}, \ldots, y_{d-1}\right)$ to the boundary, with $|y|=1$, noting that for any $\varepsilon \in \mathbb{R}, x \pm \varepsilon y \in \partial \Omega$. Then, we deduce that, for $\varepsilon>0$ sufficiently small,

$$
\frac{\phi(x+\varepsilon y)-2 \phi(x)+\phi(x-\varepsilon y)}{\varepsilon^{2}} \leq 0,
$$

where we have used the fact that $\left(v_{c}\right)_{*}(x \pm \varepsilon y)-\phi(x \pm \varepsilon y) \geq\left(v_{c}\right)_{*}(x)-\phi(x)$ whenever $\varepsilon$ is small enough, and that $\left(v_{c}\right)_{*}(x \pm \varepsilon y)=\left(v_{c}\right)_{*}(x)$ since $\left(v_{c}\right)_{*} \equiv-c$ on $\partial \Omega$. Therefore, taking the limit $\varepsilon \rightarrow 0$, we deduce from (2.2) that the second-order directional derivative $\left(y \otimes y^{\top}\right): D^{2} \phi(x) \leq 0$. Note that the matrix $B_{y}:=y \otimes y^{\top}$ belongs to the set $\mathcal{S}_{1}$ appearing in (1.2), since $B_{y}$ is positive semi-definite and has trace equal to $|y|^{2}=1$ (recall that $y$ was chosen as a unit vector). Therefore, using the definition of $H\left(D^{2} \phi(x)\right)$ from (1.2), we see that $H\left(D^{2} \phi(x)\right) \geq-B_{y}: D^{2} \phi(x) \geq 0$, and hence

$$
F^{*}\left(D^{2} \phi(x),\left(v_{c}\right)_{*}(x), x\right)=\max \left\{H\left(D^{2} \phi(x)\right),\left(v_{c}\right)_{*}(x)\right\} \geq 0,
$$

as required by (2.1). Hence $v_{c}$ is also a viscosity supersolution and thus a viscosity solution of (1.2).

Remark 2.2. In Proposition 2.1, we considered negative perturbations on the boundary, i.e. $v_{c}(x)=-c$, with $c>0$. For the case of positive perturbations, i.e. $v_{c}=c$, it is possible to construct test functions showing that the subsolution property does not hold.

\section{Dirichlet boundary conditions as in the User's Guide}

The definition of viscosity solution is formulated in a different way in the User's Guide [5]. There the gradient and Hessians obtained from the test functions define the jets 


$$
\begin{aligned}
& J^{2,+} u(x):=\left\{\left(D \phi(x), D^{2} \phi(x)\right): \phi \in C^{2} \text { and } u-\phi \text { has local maximum at } x\right\}, \\
& J^{2,-} u(x):=\left\{\left(D \phi(x), D^{2} \phi(x)\right): \phi \in C^{2} \text { and } u-\phi \text { has local minimum at } x\right\} .
\end{aligned}
$$

These jets may no be rich enough to replace the notion of the classical gradient and Hessian in the proof of a comparison principle in [5], which is why one considers the closures

$$
\begin{aligned}
& \bar{J}_{\bar{\Omega}}^{2,+} u(x):=\left\{(p, X) \in \mathbb{R}^{d} \times \mathcal{S}: \exists\left(x_{n}, p_{n}, X_{n}\right) \in \bar{\Omega} \times \mathbb{R} \times \mathcal{S}\right. \text { so that } \\
&\left.\left(p_{n}, X_{n}\right) \in J^{2,+} u\left(x_{n}\right) \text { and }\left(x_{n}, u\left(x_{n}\right), p_{n}, X_{n}\right) \rightarrow(x, u(x), p, X)\right\}, \\
& \bar{J}_{\bar{\Omega}}^{2,-} u(x):=\left\{(p, X) \in \mathbb{R}^{d} \times \mathcal{S}: \exists\left(x_{n}, p_{n}, X_{n}\right) \in \bar{\Omega} \times \mathbb{R} \times \mathcal{S}\right. \text { so that } \\
&\left.\left(p_{n}, X_{n}\right) \in J^{2,-} u\left(x_{n}\right) \text { and }\left(x_{n}, u\left(x_{n}\right), p_{n}, X_{n}\right) \rightarrow(x, u(x), p, X)\right\},
\end{aligned}
$$

which 'inherit' nearby gradients and Hessians.

In line with Example 1.11, Definition 7.4 and equation (7.24) of [5], we keep the above definitions of $F, F_{*}$ and $F^{*}$. We say that a function $v$ is a viscosity subsolution of the boundary value problem if $u$ is upper semi-continuous on $\bar{\Omega}$ and

$$
F_{*}(A, v(x), x) \leq 0 \quad \forall(A, p) \in \bar{J}_{\bar{\Omega}}^{2,+} v(x) .
$$

Similarly $v$ is a viscosity supersolution whenever $v$ is lower semi-continuous on $\bar{\Omega}$ and

$$
F^{*}(A, v(x), x) \geq 0 \quad \forall(A, p) \in \bar{J}_{\bar{\Omega}}^{2,-} v(x) .
$$

Consequently, there are two differences with the Barles-Souganidis definition:

(a) The equation is tested with a larger set of derivatives as a result of the closure of the semi-jets.

(b) Both $u$ and $v$ are assumed to be semi-continuous, rather than taking their lower and upper semi-continuous envelopes.

The functions $v_{c}$ from Proposition 2.1, which are lower semi-continuous by definition, are not affected by the closure of the jets (a) in the sense that the above arguments from the previous section related to the supersolution property of $v_{c}$ remain valid without change.

However, the requirement of semi-continuity (b) means that now, the functions $v_{c}$ do not qualify as subsolutions, (and thus are not viscosity solutions) in the sense of [5].

Thus in this case the set of viscosity solutions using the definition from [4] does not coincide with the set of solutions from [5]. More broadly, this raises the question of 


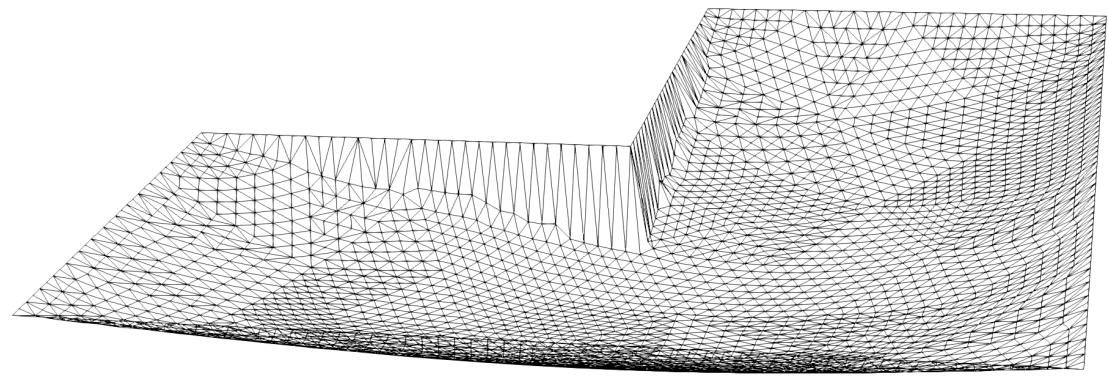

Figure 1. Numerical solution of (1.2) on an L-shaped domain with homogeneous boundary conditions and $f \equiv 1$.

the applicability of comparison principles such as [5, Theorem 7.9] when attempting to prove the convergence of numerical methods as done in [4].

\section{Dirichlet boundary conditions in the classical sense}

As in [5, Definition 2.2] we now say that a function $u$ is called a viscosity subsolution (resp. supersolution) of (1.2) if $u \in \operatorname{USC}(\Omega)$ (resp. $u \in \operatorname{LSC}(\Omega)$ ) and if for all $\varphi \in$ $C^{2}(\Omega)$ such that $u-\varphi$ has a local maximum (resp. minimum) at $x \in \Omega$ we have

$$
F\left(D^{2} \varphi(x), \nabla \varphi(x), u(x), x\right) \leq 0
$$

(resp. $F\left(D^{2} \varphi(x), \nabla \varphi(x), u(x), x\right) \geq 0$ ). In this case, the boundary conditions are not part of the definition of the viscosity solution of the equation, but are instead understood in the classical pointwise sense:

$$
u(x)=0 \quad \forall x \in \partial \Omega .
$$

This is the setting of the comparison principle [5, Theorem 3.3]. It is then clear that the functions $v_{c}$ from Proposition 2.1 are not viscosity solutions satisfying a homogeneous Dirichlet boundary conditions in the classical sense as we assume $c>0$. This shows how the set of viscosity sub- and supersolutions can then potentially differ between all three definitions described so far.

\section{An example on a bounded domain}

In the case of bounded, convex domains $\Omega$, Lemma 3.6 in [8], in the spirit of [5, Section 5.C], states that if $u$ is a subsolution and $v$ is a supersolution of (1.2) and crucially if 


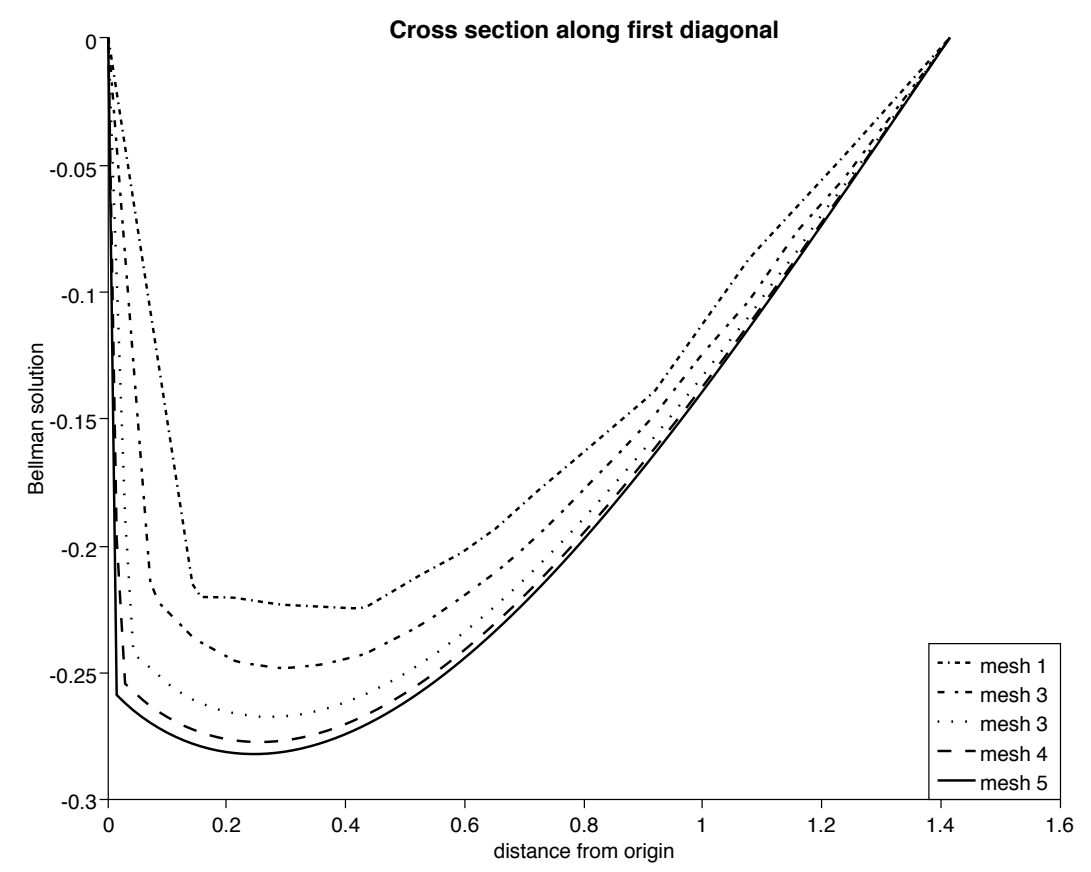

Figure 2. Cross sections of the numerical solution along the first diagonal $x_{1}=x_{2}$.

$u \leq v$ on $\partial \Omega$, then $u \leq v$ on $\bar{\Omega}$. Hence a viscosity solution that satisfies the boundary conditions in a pointwise sense is necessarily unique, if it exists.

The existence and uniqueness of viscosity solutions holds with classical boundary conditions on strictly convex domains, noting that Perron's theorem requires not only a comparison principle, but also the construction of a sub- and a supersolution, which is in full generality not possible if $\Omega$ is not strictly convex.

In fact, if the domain $\Omega$ is not strictly convex or not convex at all, then existing proofs of well-posedness $[6,7]$ require additional regularity of $\Omega$ and, in the case of non-homogeneous boundary conditions, of the boundary data. In addition the boundary data needs to be the restriction of a convex function. For the closely related problem of prescribed Gaussian curvature, Bakelman [1] imposes Dirichlet boundary conditions in terms of the border operator of convex functions, which may be viewed as leading to multi-valued solutions. A connection between $[1,6,7]$ and the Bellman formulation (1.2) is made in [10].

We highlight that the construction of a stable, monotone, consistent scheme together with the successful application of a Barles-Souganidis theorem amounts to the proof of the existence of a unique viscosity solution. Therefore one would not expect that a straightforward application of the Barles-Souganidis argument is possible on nonstrictly convex $\Omega$, unless assumptions akin to $[1,6,7]$ enter the construction. 
Indeed, it is interesting to pinpoint at which step the argument in [8] breaks down if $\Omega$ is not a strictly convex domain. Lemma 6.4 of [8] shows how, on strictly convex domains, the upper and lower semi-continuous envelopes of the numerical solutions in the small-mesh limit satisfy the classical boundary conditions; this argument relies on the existence of certain test functions, for which the strict convexity of the domain is needed.

To illustrate how on non-convex domains, the numerical solutions need not satisfy the boundary conditions in the classical sense in the small mesh limit, we shall therefore consider the scheme of [8] for (1.2) on the L-shape domain

$$
\Omega=[(0,1) \times(-1,1)] \cup[(-1,1) \times(0,1)],
$$

noting that the existence and uniqueness of numerical solutions also hold on nonconvex domains. A numerical solution is depicted in Figure 1 while Figure 2 shows the cross sections on $\left\{\left(x_{1}, x_{2}\right) \in \Omega: x_{1}=x_{2}\right\}$ of the numerical solutions over several levels of refinement, where mesh 1 is the coarsest with 328 degrees of freedom while mesh 5 has 83968 DoFs. The figures illustrate how a mesh-dependent boundary layer appears in the vicinity of the re-entrant corner. Thus it is reasonable to expect that the lower semi-continuous envelope

$$
\underline{u}(x):=\liminf _{\substack{y \rightarrow x \\ h \rightarrow 0}} u_{h}(y), \quad \forall x \in \bar{\Omega},
$$

of the sequence $\left(u_{h}\right)_{h}$ of numerical solutions will not satisfy the boundary conditions in the classical sense, so that the above mentioned comparison principle may not be used to determine the convergence of the numerical method and to guarantee existence of the viscosity solution.

\section{Mixed Dirichlet-Neumann boundary conditions as in the Barles-Souganidis theorem}

We now show some generalisations of the example of section 2 to problems with mixed boundary conditions on bounded convex domains in order to highlight some further subtleties and challenges of treating the boundary conditions in a generalised sense. We therefore return to the definition of viscosity sub- and supersolutions of [4], as detailed in section 2 .

Consider the unit square domain $\Omega=(0,1)^{2}$ in two space dimensions, and consider the simple Monge-Ampère equation (1.2) with mixed Dirichlet-Neumann boundary conditions 


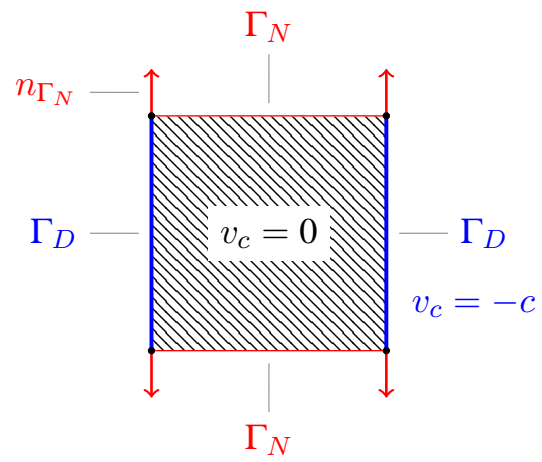

Figure 3. Construction of the viscosity solutions $v_{c}$ in Proposition 6.1.

$$
\begin{aligned}
H\left(D^{2} u\right)=0 & & \text { in } \Omega, \\
u=0 & & \text { on } \Gamma_{D}, \\
\nabla u \cdot n=0 & & \text { on } \Gamma_{N},
\end{aligned}
$$

where $H(\cdot)$ is as in (1.2), where $\Gamma_{D}=\left\{x=\left(x_{1}, x_{2}\right) \in \partial \Omega, x_{1} \in\{0,1\}, x_{2} \in\right.$ $(0,1)\}$ is composed of the left and right faces of $\partial \Omega$ (which are open relative to $\partial \Omega$ ), and $\Gamma_{N}=\left\{x=\left(x_{1}, x_{2}\right) \in \partial \Omega, x_{1} \in(0,1), x_{2} \in\{0,1\}\right\}$ is composed of the top and bottom open faces of $\partial \Omega$. Furthermore we introduce $\overline{\Gamma_{D}}$ the closure of $\Gamma_{D}$, and we note that $\overline{\Gamma_{D}}$ and $\Gamma_{N}$ partition $\partial \Omega$.

To formalize the definition of the viscosity sub- and super-solutions, we define the operator $B: \mathbb{R}^{d} \times \mathbb{R} \times \partial \Omega \rightarrow \mathbb{R}$ by

$$
B(p, r, x):- \begin{cases}r=0 & \text { if } x \in \overline{\Gamma_{D}}, \\ p \cdot n_{\Gamma_{N}}=0 & \text { if } x \in \Gamma_{N},\end{cases}
$$

where $n_{\Gamma_{N}}$ is the unit outward normal on $\Gamma_{N}$, which in this example is simply given by $n_{\Gamma_{N}}=(0,1)$ when $x_{2}=1$, and $n_{\Gamma_{N}}(0,-1)$ when $x_{2}=0$.

The lower and upper envelopes of $B$ are given by

$$
B_{*}(p, r, x):= \begin{cases}B(p, r, x) & : x \in \Gamma_{D} \cup \Gamma_{N}, \\ \min \left\{r, p \cdot n_{\Gamma_{N}}\right\} & : x \in \partial \Omega \backslash\left(\Gamma_{D} \cup \Gamma_{N}\right)\end{cases}
$$

and

$$
B^{*}(p, r, x):= \begin{cases}B(p, r, x) & : x \in \Gamma_{D} \cup \Gamma_{N}, \\ \max \left\{r, p \cdot n_{\Gamma_{N}}\right\} & : x \in \partial \Omega \backslash\left(\Gamma_{D} \cup \Gamma_{N}\right) .\end{cases}
$$

Following [4] and [5, Section 7.B], a locally bounded function $v$ is called a viscosity 
subsolution of the boundary value problem (6.1) if

$$
F_{*}\left(D^{2} \phi(x), \nabla \phi(x), v^{*}(x), x\right) \leq 0
$$

for all $\phi \in C^{2}(\bar{\Omega})$ such that $v^{*}-\phi$ has a local maximum at $x \in \bar{\Omega}$, where $F_{*}$ is defined by

$$
F_{*}(A, p, w, x)= \begin{cases}H(A) & : x \in \Omega, \\ \min \left\{H(A), B_{*}(p, w, x)\right\} & : x \in \partial \Omega .\end{cases}
$$

Analogously, $v$ is a viscosity supersolution of (6.1) whenever

$$
F^{*}\left(D^{2} \phi(x), v_{*}(x), x\right) \geq 0
$$

for all $\phi \in C^{2}(\bar{\Omega})$ such that $v_{*}-\phi$ has a local minimum at $x \in \bar{\Omega}$, where $F^{*}$ is given by

$$
F^{*}(A, w, x)= \begin{cases}H(A) & : x \in \Omega, \\ \max \left\{H(A), B^{*}(p, w, x)\right\} & : x \in \partial \Omega .\end{cases}
$$

It is clear that the function $u \equiv 0$ is a viscosity solution of the boundary value problem (6.1). However, we show in Proposition 6.1 below that again uniqueness of the viscosity solution fails due to the lack of a comparison principle.

Proposition 6.1. For a fixed but arbitrary constant $c>0$, let the locally bounded function $v_{c}$ be defined by $v_{c}=0$ on $\Omega \cup \Gamma_{N}$ and $v_{c}=-c$ on $\overline{\Gamma_{D}}$. Then $v_{c}$ is a viscosity solution of (6.1).

Proof. The upper envelope $\left(v_{c}\right)^{*} \equiv 0$ in $\bar{\Omega}$, so we see that $v_{c}$ is a subsolution. To show the supersolution property, consider a function $\phi \in C^{2}(\bar{\Omega})$ such that $\left(v_{c}\right)_{*}-\phi$ has a local minimum at $x \in \bar{\Omega}$. First, it is clear that (6.2) holds whenever $x \in \Omega$ is an interior point or when $x \in \Gamma_{N}$ is a 'Neumann' boundary point. It remains only to consider 'Dirichlet' points $x \in \Gamma_{D}$ and corner points $x \in \partial \Omega \backslash\left(\Gamma_{N} \cup \Gamma_{D}\right)$.

If $x \in \Gamma_{D}$ is a 'Dirichlet' point, i.e. $x=\left(x_{1}, x_{2}\right)$ with $x_{1} \in\{0,1\}$ and $x_{2} \in(0,1)$, then we can follow the same argument used in the proof of Proposition 2.1 to deduce that $\partial_{x_{2} x_{2}}^{2} \phi(x) \leq 0$ and hence that $H\left(D^{2} \phi(x)\right) \geq 0$. This implies that (6.2) holds whenever $x \in \Gamma_{D}$.

The only remaining case is when $x$ is a corner point, i.e. $x=\partial \Omega \backslash\left(\Gamma_{N} \cup \Gamma_{D}\right)$.

For this case, we note that for $\varepsilon>0$ sufficiently small, $x-\varepsilon n_{\Gamma_{N}} \in \Gamma_{D}$ since $n_{\Gamma_{N}}=$ $\pm(0,1)$ is the outward normal for the 'Neumann' part of the boundary. Therefore, we deduce that, for all $\varepsilon>0$ sufficiently small,

$$
\frac{\phi(x)-\phi\left(x-\varepsilon n_{\Gamma_{N}}\right)}{\varepsilon} \geq 0,
$$


where we have used the facts that $\left(v_{c}\right)_{*}\left(x-\varepsilon n_{\Gamma_{N}}\right)-\phi\left(x-\varepsilon n_{\Gamma_{N}}\right) \geq\left(v_{c}\right)_{*}(x)-\phi(x)$ for $\varepsilon>0$ sufficiently small and that $\left(v_{c}\right)_{*}\left(x-\varepsilon n_{\Gamma_{N}}\right)=v_{c}(x)=-c$.

Therefore, taking the limit $\varepsilon \rightarrow 0$ in (6.3) gives $\nabla \phi(x) \cdot n_{\Gamma_{N}} \geq 0$, and hence $B^{*}\left(\nabla \phi(x),\left(v_{c}\right)_{*}(x), x\right)=\max \left\{\nabla \phi(x) \cdot n_{\Gamma_{N}},\left(v_{c}\right)_{*}(x)\right\} \geq 0$. Thus we find that (6.2) is satisfied in the case where $x$ is a corner point. Hence $v_{c}$ is also a viscosity supersolution and thus a viscosity solution of (6.1).

\section{Bibliography}

[1] I.J. Bakelman. Generalized elliptic solutions of the Dirichlet problem for $n$-dimensional Monge-Ampère equations.Proc. of Symposia in Pure Math., 45:73-102, 1986.

[2] G. Barles, G. Burdeau.The Dirichlet problem for semilinear second-order degenerate elliptic equations and applications to stochastic exit time control problems.Comm. in PDE, 20(1-2):129-179, 1995.

[3] G. Barles, B. Perthame.Exit time problems in optimal control and vanishing viscosity method.SIAM J. Control Optim., 26(5):1133-1148, 1988.

[4] G. Barles, P.E. Souganidis.Convergence of approximation schemes for fully nonlinear second order equations.Asymptotic Anal., 4(3):271-283, 1991.

[5] M.G. Crandall, H. Ishii, P.-L. Lions.User's guide to viscosity solutions of second order partial differential equations.Bull. Amer. Math. Soc., 27(1):1-67, 1992.

[6] B. Guan, J. Spruck.Boundary-value problems on $S^{n}$ for surfaces of constant Gauss curvature.Annals of Mathematics. 138:601-624, 1993.

[7] B. Guan.The Dirichlet problem for Monge-Ampère equations in non-convex domains and spacelike hypersurfaces of constant Gauss curvature.Trans. Amer. Math. Soc. 350:49554971, 1998.

[8] X. Feng, M. JensenConvergent semi-Lagrangian methods for the Monge-Ampère equation on unstructured grids.SIAM J. Num. Anal., 55(2):691-712, 2017.

[9] C.E. Gutiérrez.The Monge-Ampère equation. Birkhäuser, 2001.

[10] M. Jensen. Numerical solution of the simple Monge-Ampère equation with non-convex Dirichlet data on non-convex domains. arXiv 1705.04653, 2017.

[11] N.V. Krylov. Nonlinear elliptic and parabolic equations of the second order. Springer, 1987.

[12] H.M. Soner. Optimal control with state-space constraint I.SIAM J Control Optim., 24:552-561, 1986.

[13] H.M. Soner.Optimal control with state-space constraint II.SIAM J Control Optim., 24:1110-1122, 1986. 


\section{Author information}

Max Jensen, Department of Mathematics, University of Sussex, Brighton BN1 9QF, England. E-mail: m.jensen@sussex.ac.uk

Iain Smears, Department of Mathematics, University College London, 25 Gordon Street, London WC1E 6BT, England.

E-mail: i.smears@ucl.ac.uk 